Case Report

\title{
Multiple Coronary Artery Microfistulas in a Girl with Kleefstra Syndrome
}

\author{
Euthymia Vargiami, ${ }^{1}$ Athina Ververi, ${ }^{1}$ Hamda Al-Mutawa, ${ }^{2}$ Georgia Gioula, ${ }^{3}$ \\ Spyridon Gerou, ${ }^{1}$ Fotios Rouvalis, ${ }^{1}$ Marios Kambouris, ${ }^{4}$ and Dimitrios I. Zafeiriou ${ }^{1}$ \\ ${ }^{1} 1 s t$ Department of Pediatrics, Aristotle University of Thessaloniki, 54642 Thessaloniki, Greece \\ ${ }^{2}$ Shafallah Medical Genetics Center, Doha, Qatar \\ ${ }^{3}$ 2nd Department of Microbiology, Aristotle University of Thessaloniki, 54124 Thessaloniki, Greece \\ ${ }^{4}$ Pathology-Genetics, Sidra Medical \& Research Center, Doha, Qatar \\ Correspondence should be addressed to Athina Ververi; athenaververis@yahoo.com
}

Received 11 October 2015; Accepted 29 February 2016

Academic Editor: Mohnish Suri

Copyright (C) 2016 Euthymia Vargiami et al. This is an open access article distributed under the Creative Commons Attribution License, which permits unrestricted use, distribution, and reproduction in any medium, provided the original work is properly cited.

Kleefstra syndrome is characterized by hypotonia, developmental delay, dysmorphic features, congenital heart defects, and so forth. It is caused by 9q34.3 microdeletions or EHMT1 mutations. Herein a 20-month-old girl with Kleefstra syndrome, due to a de novo subterminal deletion, is described. She exhibits a rare and complex cardiopathy, encompassing multiple coronary artery microfistulas, VSD/ASD, and PFO.

\section{Introduction}

Kleefstra syndrome (KS) is a recently identified entity characterized by hypotonia, developmental delay, and distinctive facial appearance. Other common features include congenital heart and urogenital defects, epilepsy, microcephaly, and behavioral abnormalities. KS is caused by either a microdeletion of $9 \mathrm{q} 34.3$ or a mutation in the euchromatin histone methyltransferase 1 (EHMT1) gene in the same region. Deletions account for $80 \%$ of cases and range from $0.04 \mathrm{Mb}$ to cytogenetically visible abnormalities [1]. The critical region of the syndrome encompasses EHMT1, which is reported as the culprit gene for the syndrome $[2,3]$. The prevalence of KS is still unknown; however, more than 100 individuals have already been described. Despite the wide variety in patients' deletion size, phenotype-genotype correlations are still weak, providing extra support for the major role of EHMT1.

Herein a 20-month-old girl with KS, due to a subterminal de novo deletion, is described. Although her phenotype is highly typical of KS, she exhibits a rather rare and complex cardiopathy with multiple coronary artery microfistulas, ventricular/atrial septal defects, and patent ductus arteriosus. Coronary anomalies have not been previously described in
KS. The girl's clinical presentation, as well as the possible mechanism underlying her cardiac defect, is discussed.

\section{Case Presentation}

The patient is the only child of healthy, nonconsanguineous parents of Caucasian origin. Pregnancy was complicated by high rupture of membranes 8 weeks prior to delivery. She was delivered at 37 weeks by cesarean section, due to mother's past history of spinal surgery. During the 4th hour of life she developed transient tachypnea and was admitted to the NICU. On the 5th day she manifested poor feeding. She was discharged after 2 weeks in good condition.

Regular pediatric follow-up at three months revealed a 3/6 systolic heart murmur. Subsequent echocardiography demonstrated multiple coronary artery microfistulas, originating from the left main coronary artery and draining into the left ventricle (Figure 1(a)). The latter was marginally hypertrophic but preserved normal contractility.

The left coronary artery was mildly dilated with an ostial diameter of $3.8 \mathrm{~mm}$. Additionally, the patient manifested multiple small ventricular septal defects, as well as haemodynamically unsignificant patent ductus arteriosus and patent 


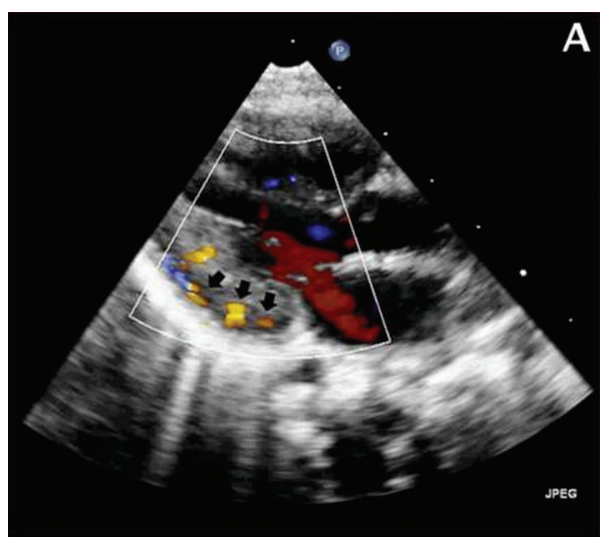

(a)

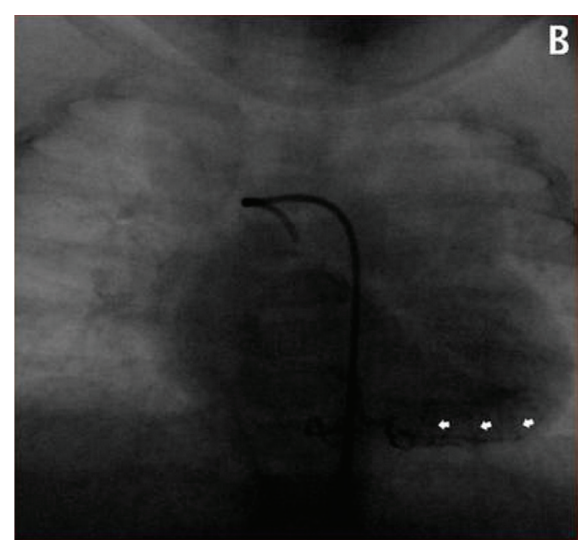

(b)

FIGURE 1: Multiple coronary artery microfistulas, originating from the left main coronary artery and draining into the left ventricle, as depicted in echo (a) and angiogram (b) (black and white arrows, resp.).

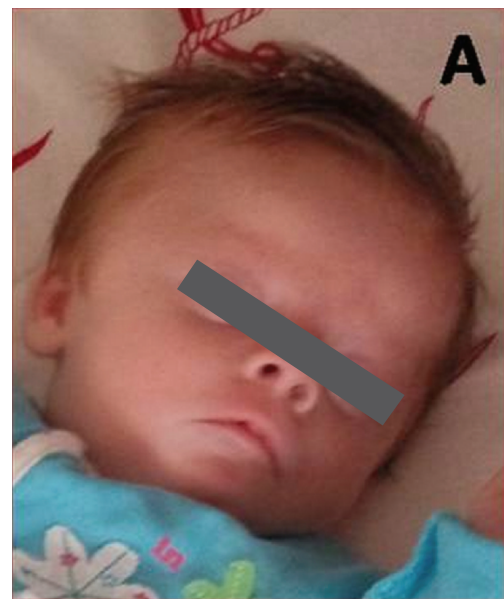

(a)

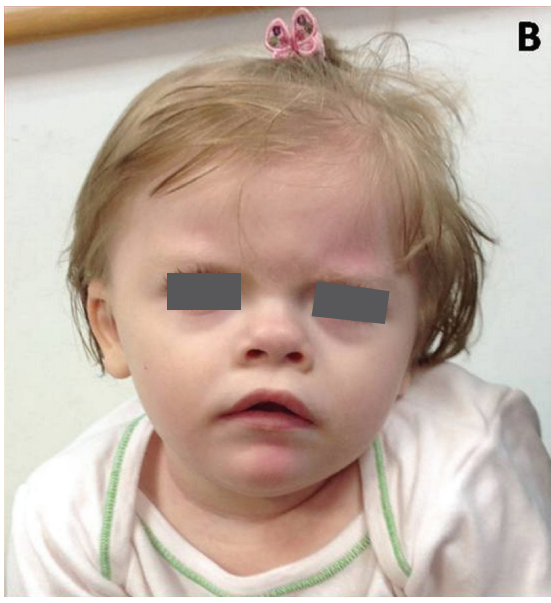

(b)

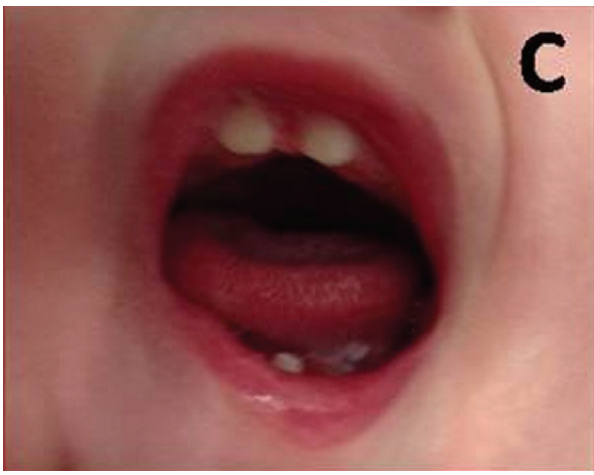

(c)

FIGURE 2: Facial profile of the patient at the age of 20 days (a) and 20 months (b); teeth anomalies at the age of 20 months (c).

foramen ovale. The valves were normal. An angiogram performed at 4 months of age confirmed the above findings (Figure 1(b)).

Conclusively, the patient exhibited a complex cardiopathy, encompassing multiple coronary artery microfistulas, mild dilatation of the left coronary artery, ventricular and atrial septal defects, and patent ductus arteriosus. Nevertheless, she was asymptomatic and required no pharmaceutical or interventional therapy.

Apart from the cardiopathy, additional clinical findings included dysmorphic features (hypertelorism, midface hypoplasia, short nose with upturned nostrils, tented upper lip, downturned corners of mouth, high arched palate, teeth anomalies, and clinodactyly of the 4th and 5th toes bilaterally), hypotonia, significant skin laxity, joint hypermobility, psychomotor delay, and progressive microcephalybrachycephaly since the age of 4 months (Figure 2). Due to the constellation of findings, molecular SNP karyotyping was performed, using Illumina Human OmniExp-12 v2 BeadChips microarray technology at a median resolution of
$4 \mathrm{~Kb}$. The analysis revealed a microdeletion of $0.55 \mathrm{Mb}$ on chromosome 9q34.3 (139.518.965-141.066.491), encompassing the critical region for KS. Parental analysis confirmed the de novo origin of the deletion.

Subsequent investigation at the age of 10 months included brain MRI, electroencephalogram, and auditory/visual evoked potentials, which were all normal. Due to the presence of microcephaly, the patient underwent cranial 3D-CT, which revealed fusion of both coronal sutures. At the age of 14 months she had an episode of generalized tonicclinic seizures, followed by paroxysmal activity on postictal electroencephalograms. She was started on valproate and has been so far free of episodes.

At the age of 20 months, the patient has moderate-severe psychomotor delay with hypotonia, poor visual and auditory responsiveness, and a limited vocabulary of 1-2 words. She has moderate head control and poor trunk control but can sit with support. Her head circumference is below the 3 rd centile $(-2.5 \mathrm{SD})$ and her weight and height are at the $97 \mathrm{th}$ and 75th centiles, respectively. She is having physiotherapy 
TABLE 1: Summary of main features in 91 reported patients with $9 \mathrm{q} 34.3$ deletion [1] and in the index patient.

\begin{tabular}{|c|c|c|}
\hline Clinical features & $\%$ in 91 patients & Present in proband \\
\hline \multicolumn{3}{|l|}{ Growth parameters } \\
\hline High birth weight & 9 & - \\
\hline Microcephaly & 50 & + \\
\hline Short stature & 32 & - \\
\hline Overweight (BMI > 25) & 28 & - \\
\hline $\mathrm{DD} / \mathrm{ID}$ & 100 & + \\
\hline Heart defect & 41 & + \\
\hline Renal anomaly (including vesicoureteral reflux) & 12 & - \\
\hline $\begin{array}{l}\text { Behavioral/psychiatric problems (including autistic features, attention deficit problems, } \\
\text { self-mutilation, aggressive and emotional outbursts/crises, and severe sleep disturbance) }\end{array}$ & $54 \%$ & - \\
\hline Recurrent infections & 26 & - \\
\hline Hearing deficit & 23 & - \\
\hline Gastroesophageal reflux & 19 & - \\
\hline Epilepsy & 36 & + \\
\hline Anomalies on brain imaging & 58 & - \\
\hline Musculoskeletal anomalies (including joint hypermobility, scoliosis, and club foot) & 25 & + \\
\hline Teeth anomalies & $<10 \%$ & + \\
\hline Hyperelastic skin & 0 & + \\
\hline
\end{tabular}

and occupational therapy. As far as her cardiopathy is concerned, the condition remains asymptomatic with the majority of microfistulas gradually resolving in a spontaneous way.

\section{Discussion}

A 20-month-old girl with KS and an atypical cardiac manifestation with coronary artery microfistulas is herein reported.

Coronary artery microfistulas have not been so far documented in either 9q34.3 deletions or EHMT1 mutations, despite the high prevalence of cardiac manifestations in the syndrome. In a cohort of 112 patients described by Willemsen et al. [1], approximately $40 \%$ manifested heart defects, mainly structural anomalies, such as ventricular and atrial septal defects, patent foramen ovale, valve and ductus arteriosus anomalies, tetralogy of Fallot, and so forth. More rare manifestations included an aberrant muscle band in the left ventricle of one individual, as well as cardiac arrhythmias in another two patients [1]. The clinical features of the cohort are summarized in Table 1.

Coronary artery fistulas (CAFs) are rare vascular anomalies presenting in $0.002 \%$ of the general population and involving either the great vessels or the cardiac chambers, mainly the right ones. Only $10 \%$ of CAFs affect the left heart structures [4], as in the index patient. CAFs are usually congenital in origin, probably resulting from the persistence of embryonic intertrabecular spaces and sinusoids [5]. Chemotactic, haemodynamic, and chemical factors have been associated with CAFs development [6], although their exact etiology remains still elusive. No genetic cause has been so far related to CAFs. There is one report of CAFs in dizygotic twins, but the authors suggested the presence of a poorly identified environmental factor during pregnancy [7].
As far as CAFs association with genetic syndromes is concerned, CAFs have been sporadically described in an infant with 22q11 deletion syndrome [8] and a man with mosaic Klinefelter syndrome [9], as well as three older patients with clinical diagnoses of Marfan [10], Goldenhar [11], and RenduOsler-Weber [12] syndromes. The latter condition is associated with 5 different genetic causes, including ENG mutations at 9q34.11 [13], which is $9 \mathrm{Mb}$ away from the critical Kleefstra region. Moreover, molecular analysis was not undertaken in that patient and, thus, neither the diagnosis nor the exact genetic type of Rendu-Osler-Weber was confirmed. The infant with 22q11 deletion manifested left ventricular noncompaction and a CAF between the left coronary artery and the right ventricle outflow tract [8], whereas the man with Klinefelter syndrome had a CAF between the left coronary artery and the right ventricle [9]. The patient with Marfan syndrome had a left CAF draining into the superior vena cava and the right superior pulmonary vein, as well as a second fistulous communication between the left coronary sinus and both atria [10]. Marfan syndrome is associated with mutations or deletions in FBN1 at 15q21.1 [14], but the index patient did not have molecular confirmation of the diagnosis. Last but not least, a CAF between the left coronary artery and the right atrium was reported in a woman with Goldenhar syndrome [11]. Goldenhar syndrome has no unique heritable cause but has been associated with chromosomal abnormalities, including pericentric inversion of chromosome 9 [46 XY inv 9 (p11; q13)] [15]. It should be noted that none of the above syndromes involve 9q34.3 deletion. Moreover, all five patients demonstrated solitary fistulous communications, in contrary to the multiple microfistulas of our patient.

The deleted region in the index patient contains 38 OMIM genes. Seven of them are disease-causing: AGPAT2, MAN1B1, GRIN1, TPRN, SLC34A3, NSMF, and EHMT1. Apart from 
EHMT1, the other six are associated with congenital lipodystrophy type 1, mental retardation, deafness, hypophosphatemic rickets, and hypogonadotropic hypogonadism. Due to their high haploinsufficiency scores (>60\%) [16], AGPAT2, $T P R N, S L C 34 A 3$, and NSMF are highly unlikely to contribute to the patient's phenotype. MAN1B1 and GRIN1, which are both associated with intellectual deficits have low indexes of $30.3 \%$ and $33.4 \%$, respectively [16]. However, neither these, nor the rest of the 38 deleted genes, have been related to coronary abnormalities so far.

Heart anomalies in KS are equally present in patients carrying a 9q34.3 deletion or an intragenic EHMT1 mutation [1]. EHMT1 deficiency is, therefore, strongly implicated in the cardiac phenotype of the syndrome. It should be noted, however, that two patients with MAN1B1 mutations exhibited dilatation of the aortic root and ventricular septal defect, respectively, along with mental retardation, hypotonia, mild dysmorphic features, joint hypermobility, and skin laxity. The latter feature was prominent in our patient, albeit not typical of KS. It is also noteworthy that neither these patients nor ours manifested disruption of COL5A1 at $9 \mathrm{q} 34.3$, which is the culprit gene for classic Ehlers-Danlos syndrome and, also, linked to the hypermobility in KS. MAN1B1 deficiency is currently associated with autosomal recessive intellectual disability. Nevertheless, the two patients were unresolved cases of congenital disorders of glycosylation (CDG) type II and were found to carry MAN1B1 mutations through exome sequencing. Five other patients with CDG II and MAN1B1 mutations have been so far identified. All patients carried either homozygous or compound heterozygous mutations [17]. Heart involvement has been often described in patients with CDG, ranging from cardiomyopathy to conotruncal defects [18]. Both manifestations have been recently studied in mouse models of the disease $[19,20]$. One proposed mechanism for structural heart defects involves deletion of $\mathrm{N}$-cadherin in neural crest cells, which, subsequently, fail to participate in embryonic cardiac development [19].

Unfortunately, no experimental animal model for either CAFs or cardiac manifestations in KS has yet been developed. $\mathrm{An} \mathrm{EHMT1}^{+-}$mouse model has only recently recapitulated the developmental features of the core KS phenotype [21]. Future experimental work with animal models, as well as identification of new patients with KS, will probably dissect the cardiac features and identify the culprit gene, whether it is EHMT1 alone or EHMT1 in conjunction with MAN1B1 and others. As far as CAFs are concerned, this is their first description in KS and, thus, a definitive link cannot be established. It can be, however, supported that cardiac manifestations, either common or rare, warrant KS investigation in unresolved cases with syndrome-indicative phenotypes.

\section{Competing Interests}

The authors declare that they have no competing interests.

\section{References}

[1] M. H. Willemsen, A. T. Vulto-Van Silfhout, W. M. Nillesen et al., "Update on Kleefstra syndrome," Molecular Syndromology, vol. 2, no. 3-5, pp. 202-212, 2012.
[2] T. Kleefstra, H. G. Brunner, J. Amiel et al., "Loss-of-function mutations in Euchromatin histone methyl transferase 1 (EHMT1) cause the 9q34 subtelomeric deletion syndrome," American Journal of Human Genetics, vol. 79, no. 2, pp. 370-377, 2006.

[3] T. Kleefstra, W. A. Van Zelst-Stams, W. M. Nillesen et al., "Further clinical and molecular delineation of the $9 \mathrm{q}$ subtelomeric deletion syndrome supports a major contribution of EHMT1 haploinsufficiency to the core phenotype," Journal of Medical Genetics, vol. 46, no. 9, pp. 598-606, 2009.

[4] C. V. Mangukia, "Coronary artery fistula," Annals of Thoracic Surgery, vol. 93, no. 6, pp. 2084-2092, 2012.

[5] J. T. Davis, H. D. Allen, J. J. Wheller et al., "Coronary artery fistula in the pediatric age group: a 19-year institutional experience," The Annals of Thoracic Surgery, vol. 58, no. 3, pp. 760-763, 1994.

[6] S. A. M. Said, A. A. H. J. Thiadens, M. J. C. H. Fieren, E. J. Mejiboom, T. van der Werf, and G. B. W. E. Bennink, "Coronary artery fistulas," Netherlands Heart Journal, vol. 10, pp. 65-78, 2002.

[7] S. Caputo, N. Sorice, R. Sansone et al., "Echocardiographic diagnosis of coronary artery fistula in both dizygotic twin brothers: environmental mechanism?" Journal of Cardiovascular Medicine, 2010.

[8] H. Branton, A. E. Warren, and L. S. Penney, "Left ventricular noncompaction and coronary artery fistula in an infant with deletion 22q11.2," Pediatric Cardiology, vol. 32, no. 2, pp. 208210, 2011.

[9] K. Yoshida, T. Ryu, T. Ogata et al., "An elderly man with Klinefelter syndrome associated with hypertrophic cardiomyopathy, sick sinus syndrome, and coronary arteriovenous fistula," Japanese Circulation Journal, vol. 62, no. 3, pp. 222-224, 1998.

[10] S. Williams-Phillips, "Marfan's syndrome: pre-pubertal aortic rupture with left coronary artery aneurysms and fistulas," West Indian Medical Journal, vol. 61, no. 9, pp. 937-940, 2012.

[11] O. M. Hess, J. Steurer, N. H. Goebel, U. Kuhlmann, and H. P. Krayenbühl, "Goldenhar syndrome," Schweizerische Medizinische Wochenschrift, vol. 109, no. 1, pp. 19-23, 1979 (German).

[12] M. A. Jacob, S. B. Goyal, L. Pacifico, and D. H. Spodick, "Multiple coronary artery-left ventricular fistulas associated with hereditary hemorrhagic telangiectasia," Chest, vol. 120, no. 4, pp. 1415-1417, 2001.

[13] K. A. McAllister, K. M. Grogg, D. W. Johnson et al., "Endoglin, a TGF- $\beta$ binding protein of endothelial cells, is the gene for hereditary haemorrhagic telangiectasia type 1," Nature Genetics, vol. 8, no. 4, pp. 345-351, 1994.

[14] M. E. S. Colovati, L. R. J. Da Silva, S. S. Takeno et al., "Marfan syndrome with a complex chromosomal rearrangement including deletion of the FBN1 gene," Molecular Cytogenetics, vol. 5, no. 1, article 5, 2012.

[15] M. Stanojević, F. Stipoljev, B. Koprčina, and A. Kurjak, "Oculoauriculo-vertebral (Goldenhar) spectrum associated with pericentric inversion 9: coincidental finding or etiologic factor?" Journal of Craniofacial Genetics and Developmental Biology, vol. 20, no. 3, pp. 150-154, 2000.

[16] N. Huang, I. Lee, E. M. Marcotte, and M. E. Hurles, "Characterising and predicting haploinsufficiency in the human genome," PLoS genetics, vol. 6, no. 10, Article ID e1001154, 2010.

[17] D. Rymen, R. Peanne, M. B. Millón et al., "MAN1B1 deficiency: an unexpected CDG-II," PLoS Genetics, vol. 9, no. 12, Article ID e1003989, 2013. 
[18] E. J. Footitt, A. Karimova, M. Burch et al., "Cardiomyopathy in the congenital disorders of glycosylation (CDG): a case of late presentation and literature review," Journal of Inherited Metabolic Disease, vol. 32, supplement 1, pp. S313-S319, 2009.

[19] Y. Luo, F. A. High, J. A. Epstein, and G. L. Radice, "N-cadherin is required for neural crest remodeling of the cardiac outflow tract," Developmental Biology, vol. 299, no. 2, pp. 517-528, 2006.

[20] B. Schroen, J. J. Leenders, A. van Erk et al., "Lysosomal integral membrane protein 2 is a novel component of the cardiac intercalated disc and vital for load-induced cardiac myocyte hypertrophy," Journal of Experimental Medicine, vol. 204, no. 5, pp. 1227-1235, 2007.

[21] M. C. M. Balemans, M. Ansar, A. R. Oudakker et al., "Reduced Euchromatin histone methyltransferase 1 causes developmental delay, hypotonia, and cranial abnormalities associated with increased bone gene expression in Kleefstra syndrome mice," Developmental Biology, vol. 386, no. 2, pp. 395-407, 2014. 


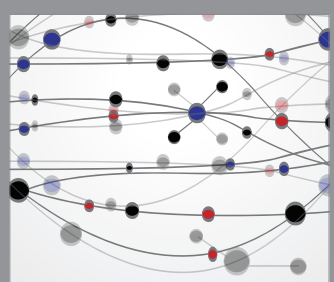

The Scientific World Journal
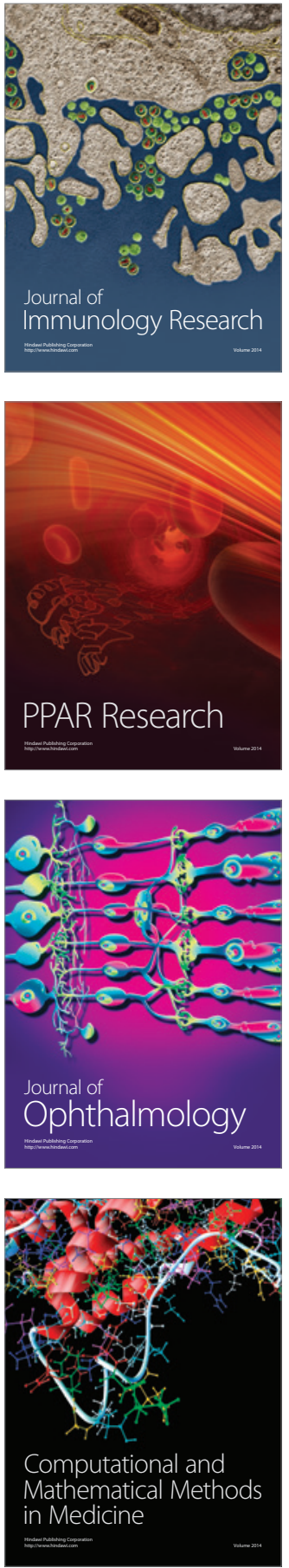

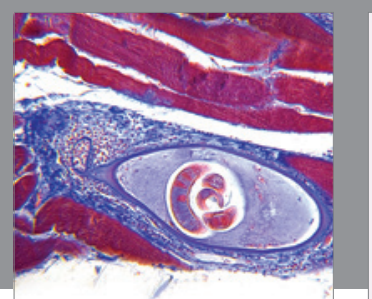

Gastroenterology Research and Practice

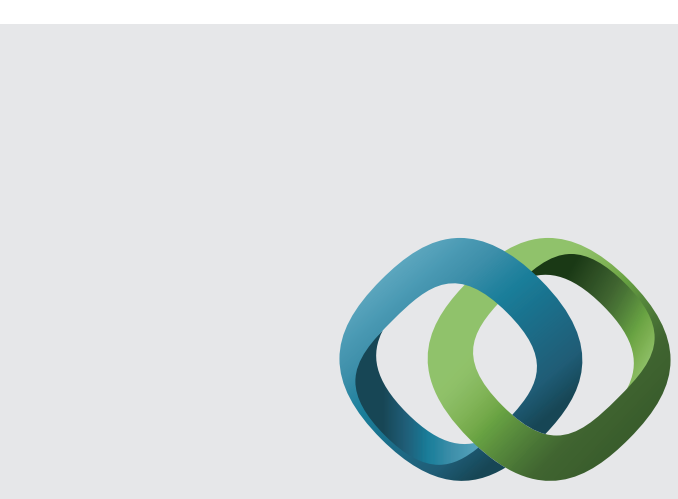

\section{Hindawi}

Submit your manuscripts at

http://www.hindawi.com
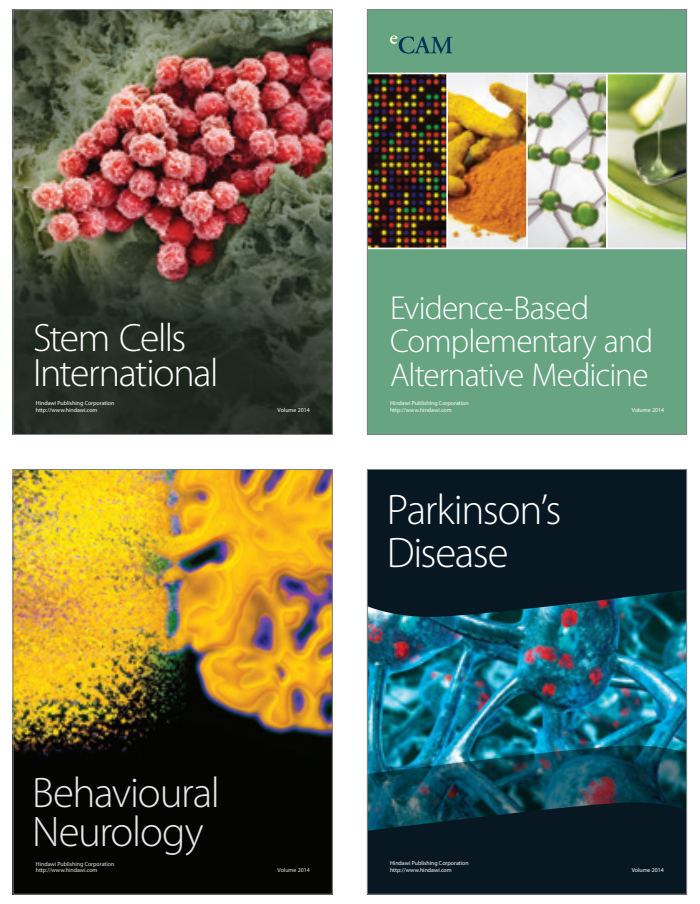
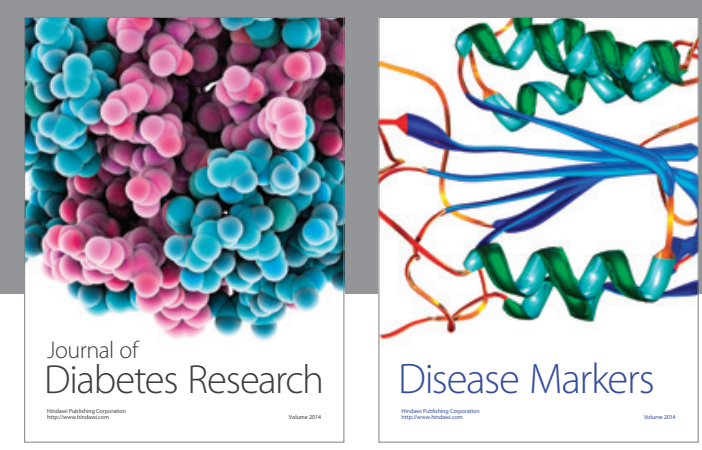

Disease Markers
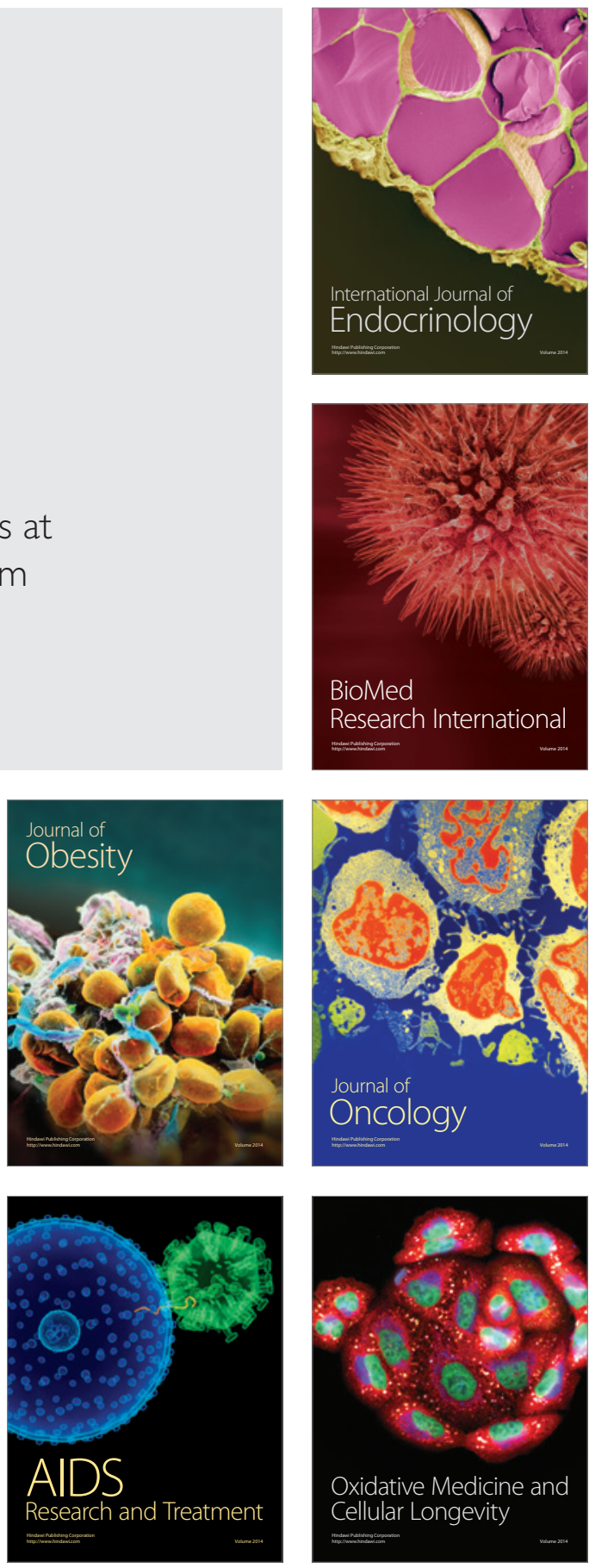